\title{
HIGHER DIMENSIONAL INDECOMPOSABLE CONNECTED SETS
}

\author{
PAUL M. SWINGLE
}

Here we wish to show the existence of indecomposable, nonclosed, connected sets, especially hereditary ones, contained densely in connected domains of $E_{n}, n>1$, or in the Hilbert cube $I_{\omega}$. We recall that: an indecomposable connected set $M$ is a connected set which is not the sum of two connected subsets, each with a different closure than that of $M$; it is also hereditary if every connected subset is an indecomposable connected set. We call a nondegenerate connected set a connexe. A closed indecomposable connexe is an indecomposable continuum.

We have shown with Hunter in [4] that there exists a hereditarily indecomposable connexe contained densely in a connected domain of $E_{2}$. Also it was shown in Theorem 4 of [4] that there exists in a solid torus of $E_{3}$ an arc-wise path, without any crossing, densely contained interior to the torus, which is an indecomposable connexe: it is the proof of this Theorem 4 we wish to modify to give the results of this paper; by an arc-wise path $P$ here we mean a set of points $P$ which is the sum of simple continuous arcs $p_{i} p_{i+1}(i=0,1,2, \cdots), p_{i}$ a point, such that $p_{0} p_{1}+p_{1} p_{2}+\cdots+p_{h-1} p_{h}$ is a simple continuous arc, for each $h$, from $p_{0}$ to $p_{h}$. Thus intuitively we may think of the indecomposable connexe $P$ as generated by an idealized particle $Q$ moving over the points of $P$, being at time $t_{i}$ at point $p_{i}$ where $\operatorname{Lim} t_{i}=\infty$ say.

To give a quicker intuitive grasp of the methods used below we wish to speak of a basic connexe densely extendable over a connected domain, which may be the interior of a torus in $E_{3}$ or its generalization in $E_{n}$ or $I_{\omega}$, to give a desired indecomposable connexe. Such a connexe, for Theorem 4 of [4] is an arc minus one end point: for if both this and the arc-wise path $P$ are considered as 1-dimensional spaces in themselves with a 1-dimensional topology, i.e. region is an arc minus end points in both, they are homeomorphic. The construction below is dependent upon an indecomposable connexe $M$ with only one composant, which is $r$-dimensional at each of its points, and this connexe is contained densely in a domain $D$. If we take a basis of connected, $r$-dimensional regions for the topology of this composant, then the basic connexe densely extendable over $D$ for $M$ is a set $W$ which is homeomorphic with this composant under a similar

Received by the editors December 18, 1956. 
topology, i.e. with a basis of connected $r$-dimensional regions. See $[3$, pp. $2-3,70]$; for fundamental definitions see $[1 ; 2 ;$ or 3$]$. For $E_{3}$ below we use a solid triangle minus one vertex for $W$, and for higher dimensional spaces $W$ will be the $r$-dimensional generalization of this, i.e. a solid $r$-triangle, or $r$-pyramid, minus one vertex $A$. This solid $r$-pyramid will be homeomorphic to an $r$-dimensional solid with center line a straight line $B A$ and with cross sections perpendicular to this consisting of the cartesian product of $(r-1)$ straight line intervals, where, if $d_{\text {: }}$ is the diameter of this cross section at $x$ on $B A$ and if $x$ approaches the $\operatorname{limit} A$, then $\operatorname{Lim} d_{x}=0$ : the solid $r$-pyramid is the sum of these cross sections for $x \in B A$, but $x \neq A$. Intuitively we may think of generating this solid $r$-pyramid by letting $x$ move from $B$ to $A$ on $B A$ carrying this cross section with it. We will call the solid triangle minus a vertex a solid 2-pyramid. By the vertex of an $r$ pyramid we will mean this missing vertex $A$.

Theorem 1. Let $D$ be a connected domain imbedded in $E_{n}, n>2$, or $I_{\omega}$ and let $W$ be a solid r-pyramid. Then there exists an indecomposable connexe $M$, with only one composant and it $r$-dimensional, densely contained in $D$ whose basic connexe densely extendable over $D$ is $W$.

ProOF. We give the proof first for $E_{3}$ and $r=2$, observing however that the fundamentals used in the proof also have equations in the higher dimensional spaces. Let $E_{3}$ be an $\left(x_{1} x_{2} x_{3}\right)$-coordinate space. We obtain this proof as a modification of that of Theorems 1 and 4 of [4]: this in turn is obtained by combining the tunneling process of Wada as used in [5, pp. 178-179, Theorem 1] for the construction of an indecomposable connexe, with the method of constructing a simple continuous arc as in $[2$, pp. $86-88$, Theorem 1] or in $[3$, p. 80 , Theorem 3.9]. In [4] we obtained our covering of $D$, which gives the simple chain of regions needed for the construction of the arc, by taking interiors of spheres as regions: here we will take instead the interiors of ellipsoids, with larger axis parallel to the $x_{1}$-axis: with center at $(0,0,0)$ the equation of the ellipsoid will be taken as $\left(x_{1} / a_{1}\right)^{2}$ $+\left(x_{2} / a_{2}\right)^{2}+\left(x_{3} / a_{3}\right)^{2}=1$, where $a_{1}>a_{2}$ or $a_{3}$.

In the proof in [4] a set of points $p_{0}, p_{1}, \cdots, p_{j}, \cdots$ is taken in $D$ in such a manner that one can have the construction below. A simple chain of regions, $C_{1}$, is taken from $p_{0}$ to $p_{1}$; a simple chain $C_{2}$ is taken which is the sum of chains from $p_{0}$ to $p_{1}$ and from $p_{1}$ to $p_{2}$; in general a simple chain $C_{i}$ is taken from $p_{0}$ to $p_{i}$, which is the sum of simple subchains from $p_{0}$ to $p_{1}$, from $p_{1}$ to $p_{2}, \cdots$, and from $p_{i-1}$ to $p_{i}$. Let $T_{i}$ be the sum of the regions of $C_{i}$; let $Z_{i}$ be the boundary of $T_{i}$ except for the part of this boundary which is also part of the boundary 
of the first and last regions of $C_{i}$ : since $Z_{i}$ is homeomorphic to a cylinder, we will call it a cylinder. The chains $C_{i}$ are taken so that the $Z_{i}(i=1,2, \cdots)$ are mutually exclusive; also the parts of $T_{i}$ and $T_{i+1}$ from $p_{h-1}$ to $p_{h}, 1 \leqq h \leqq i$, i.e. the sums of the regions of these subchains of $C_{i}$ and $C_{i+1}, T_{i}^{\prime}$ and $T_{i+1}^{\prime}$ say, are such that $T_{i}^{\prime} \supset \bar{T}_{i+1}^{\prime}$. Furthermore these chains are taken so that $\cap \bar{T}_{i}^{\prime}$ is a simple continuous arc $p_{h-1} p_{h}$. Thus, if $r_{h i}$ is the radius of the largest sphere of the chain $C_{i}$ from $p_{h-1}$ to $p_{h}, \operatorname{Lim} r_{h i}=0$. Also the chains are taken so that $p_{0} p_{1}+p_{1} p_{2}+\cdots+p_{g-1} p_{g}(g=1,2, \cdots)$ is an arc; and $M$ $=p_{0} p_{1}+p_{1} p_{2}+\cdots+p_{g-1} p_{g}+\cdots$ is dense in $D$. All of this can be done by well known methods.

We see that $M$ is an indecomposable connexe by the usual method: Suppose $M$ is the sum of the two connected subsets $H$ and $K$ neither of which has the same closure as $M$ does. Let $R_{h}$ and $R_{k}$ be regions such that $H \supset \bar{R}_{h} \cdot M, \bar{R}_{h} \cdot K=0$, and $K \supset \bar{R}_{k} \cdot M$. By the method of construction giving the above properties there exists a simple subchain of some $C_{i}$ whose end regions are in $R_{h}$ and one of whose regions is contained in $R_{k}$, but does not contain all of $R_{k} \cdot M$. Thus a subcontinuum of $\left(Z_{i}\right.$ plus the boundary of $\left.R_{h}\right)$ separates the connexe $K$, which is a contradiction, since neither $Z_{i}$ nor the boundary of $R_{h}$ contains a point of $K$.

The proof of Theorem 1 here is similar, taking ellipsoids of revolution with large axis parallel to the $x_{1}$-axis of the space, in place of the spheres in [4]: thus in [4] the cylinders $Z_{i}$ have circular cross sections, while here these cross sections are ellipses. If $a_{h i}$ is the larger semiaxis, $r_{h i}$ the smaller for the largest of these cross sections, and $b_{h i}$ is the largest semiaxis of the smallest of the cross sections of $Z_{i}$ between $p_{h-1}$ and $p_{h}$, then we take $\operatorname{Lim} r_{h i}(i \rightarrow \infty)=0, \operatorname{Lim} a_{h i}(i \rightarrow \infty)$ $=a_{h}$, and $\operatorname{Lim} b_{h i}(i \rightarrow \infty) \neq 0$. Each ellipsoid with smaller semiaxis $r_{i}$ has about its center a sphere of radius $r_{i}$. If these spheres are substituted for the ellipsoids, we may take the chains so as to get arcs as above. In order that the indecomposable connexe $M$ of Theorem 1 be dense in $D$ we must take $a_{h}$ above so that $\operatorname{Lim} a_{h}(h \rightarrow \infty)=0$. Thus, in part by the above methods, we see without great difficulty that this Theorem is true for $E_{3}$ and $r=2$. For $r>2$ in $E_{n}$ we must treat $r-1$ of the semiaxes as we did $a_{h i}$ above and the remaining as $r_{h i}$ was treated: thus for this case we use generalized ellipsoids rather than ellipsoids of revolution. Similar modifications indicate the Theorem is true for $I_{\omega}$, in spite of the intuitive nature of the argument above.

Corollary 1.1. Let $D$ be a connected domain imbedded in $E_{n}, n>2$, or in $I_{\omega}$. Then $D$ contains densely an indecomposable connexe $M$ with 
an uncountable number of composants, each of which is an arc-wise path dense in $D$.

Proof. This follows from Theorem 1 : for if $W$ is the solid $r$-pyramid with base $W^{\prime}$ and vertex $A$, one can join uncountably many of the points of $W^{\prime}$ to $A$ by straight line intervals. With the obvious $r$ dimensional topology on both $W$ and the composant of $M$ in Theorem 1 , we have a homeomorphism between these which carries these straight line intervals into the desired composants of this corollary.

Corollary 1.2. Let $D$ be a connected domain imbedded in $E_{n}, n>1$, or in $I_{\omega}$. Then $D$ contains densely a hereditarily indecomposable connexe $M$ of $r$-dimensions, $1 \leqq r<$ the dimension of the space.

Proof. For $n=2$ this is Theorem 9 of [4]. Thus let the space be as in Theorem 1 above, from which this corollary follows. Let $W$ be as in the proof of Corollary 1.1 above. Let $I$ be a hereditarily indecomposable continuum of $r$-dimensions of [6], or of [7] if $r=2$, such that $I$ is imbedded in $W+A$ of the proof above and $I$ contains both $A$ and points of the base $W^{\prime}$. Thus the homeomorphism as above which carries $W$ into the one composant of Theorem 1, carries $I$ into the set of points $M$ of this corollary, which is the desired hereditarily indecomposable connexe densely contained in $D$. We note that, if $C$ is a connexe of $M$ such that $\bar{C} \neq \bar{M}$, then $\bar{C}$ is a proper subcontinuum of the one composant of the indecomposable connexe of Theorem 1; hence here $C$ must be indecomposable. Thus the proof gives no difficulty.

\section{REFERENCES}

1. C. Kuratowski, Topologie, vols. 1, 2 Polska Akademia Nauk, Monografie Matematyszne Tom XX (1952).

2. R. L. Moore, Foundations of point set theory, Amer. Math. Soc. Colloquium Publications vol. 131932.

3. R. L. Wilder, Topology of manifolds, Amer. Math. Soc. Colloquium Publications vol. 32, 1949.

4. R. P. Hunter and P. M. Swingle, Indecomposable trajectories.

5. P. M. Swingle, Closures of types of indecomposable connexes, Proc. Amer. Math. Soc. vol. 2 (1951) pp. 178-185.

6. R. H. Bing, Higher dimensional hereditarily indecomposable continua, Trans. Amer. Math. Soc. vol. 71 (1951) pp. 267-273.

7. B. Knaster, Un continu dont tout sous-continu est indecomposable, Fund. Math. vol. 3 (1922) pp. 247-286.

The University of Miami 